\title{
Short-wavelength Spin Waves in Yttrium Iron Garnet Micro-Channels on Silicon
}

\author{
Ping Che ${ }^{1,4}$, Yan Zhang ${ }^{2}$, Chuanpu Liu ${ }^{1,3}$, Sa Tu ${ }^{1,4}$, Zhe He ${ }^{1}$, Youguang Zhang ${ }^{1}$, Zhiming Liao ${ }^{3}$, \\ Dapeng $\mathrm{Yu}^{3}$, Francesco Antonio Vetro ${ }^{4}$, Jean-Philippe Ansermet ${ }^{4}$, Weisheng Zhao ${ }^{1}$, Lei $\mathrm{Bi}^{2}$, and \\ Haiming $\mathrm{Yu}^{1,4}$ \\ ${ }^{1}$ Fert Beijing research institute, School of Electronic and Information Engineering, BDBC, Beihang University, Beijing 100191, People's \\ Republic of China \\ ${ }^{2}$ State Key Laboratory of Electronic Thin Films and Integrated Devices, University of Electronic Science and Technology of China, \\ Chengdu 610054, People's Republic of China \\ ${ }^{3}$ State Key Laboratory for Mesoscopic Physics, School of Physics, Peking University, Beijing 100871, People's Republic of China \\ ${ }^{4}$ Laboratory of Nanoscale Magnetic Materials and Magnonics, Institute of Materials, Ecole Polytechnique Fédérale de Lausanne, \\ Lausanne $\mathrm{CH}-1015$, Switzerland
}

Received......

Abstract—Yttrium iron garnet (YIG) has been widely used in spin wave studies thanks to its low Gilbert damping constant. However most of the high quality YIG films are grown on gadolinium gallium garnet (GGG) substrate, which makes it difficult to integrate with existing semiconductor technology. We show spin wave excitation in nanometer thick YIG microchannel on silicon substrate. The YIG is grown by pulsed laser deposition (PLD) technique in high-purity oxygen followed by rapid thermal annealing at $800^{\circ} \mathrm{C}$ after deposition. Using meander coplanar waveguides at submicron scale, spin waves with wavelength down to $1 \mu \mathrm{m}$ are excited. By measuring the linewidth of the spin wave reflection spectra, a Gilbert damping constant $\alpha=1.1 \times 10^{-3}$ was obtained.

Index Terms-Magnetodynamics, yttrium iron garnet film, pulsed laser deposition, coplanar waveguide, spin wave, Gilbert damping.

\section{INTRODUCTION}

The complementary metal oxide semiconductor (CMOS) for integrated circuits over six decades play an important role in nowadays information processing and computing systems. With the fabrication nodes of transistors shrunk to tens of nanometers, the heating losses and leakage current tend to be an obstacle for further development and Moore's law runs out of steam. The emerging field of magnon spintronics provides an alternative approach, using spin waves (magnons) in magnetic materials as information carriers [Grundler 2015, Chumak 2015]. Until now, wide varieties of magnetic materials have been explored for magnon spintronics, including metals and insulators. Metallic ferromagnets, such as $\mathrm{CoFeB}$ and Permalloy ( $\mathrm{Ni}_{80} \mathrm{Fe}_{20}$ ), show Gilbert damping constants of around 0.007 [Yu 2012] and 0.009 [Bauer 2015] at room temperature, respectively, which are a few orders of magnitude larger than that of bulk yttrium iron garnet $\left(\mathrm{Y}_{3} \mathrm{Fe}_{5} \mathrm{O}_{12}\right.$, YIG). Meanwhile, electron transport could not be completely avoided in metals, as the excitation and detection methods of spin waves involve microwave field. However, these issues can be completely avoided in YIG thanks to its insulating property. Thus, YIG has been regarded as one of the favorite candidates for magnon spintronics research.

YIG thin films are mainly deposited on gadolinium gallium garnet $\left(\mathrm{Gd}_{3} \mathrm{Ga}_{5} \mathrm{O}_{12}, \mathrm{GGG}\right)$, which a nonmagnetic garnets. GGG is an ideal substrate for depositing YIG because it has almost the same lattice parameter as YIG when these two garnets are in perfect lattice
[Linares 1965]. However, the difficulty of integrating GGG with silicon-based circuits maintains a barrier for applications of magnon spintronics.

In this letter, YIG thin film grown on silicon $(\mathrm{Si})$ substrate with Gilbert damping $\alpha=1.1 \times 10^{-3}$ is reported. Even though the huge mismatch of lattice constant between Si and YIG makes the Gilbert damping constant worse than YIG/GGG $\left(\alpha=8.58 \times 10^{-5}\right.$ by sputtering [Chang 2014] and $\alpha=2.3 \times 10^{-4}$ by PLD [Kelly 2013]), it has less losses than most of the metallic magnetic materials and makes the integration of magnon spintronics devices with traditional circuits possible. Coplanar waveguide (CPW), consisting of one signal line in the center of two ground lines has been used for spin wave excitation [Bao 2008, Albisetti 2016]. A specially designed meander CPW was used here to excite spin waves in YIG microchannels on Si substrate. The spin wave wavevector can be well controlled, and corresponds to a wavelength smaller by at least one order of magnitude than that of the spin waves excited by conventional microwave strips [Cornelissen 2015].

\section{EXPERIMENT}

YIG thin films were deposited on Si (100) substrates by pulsed laser deposition (PLD) techniques. A KrF excimer laser operating at $\lambda=248 \mathrm{~nm}$ wavelength was used for thin film deposition. During deposition, the frequency of the pulsed laser was $10 \mathrm{~Hz}$ and the energy density on the target surface was about $2.5 \mathrm{~J} / \mathrm{cm} 2$. The distance 
between the target and the substrate was fixed at $55 \mathrm{~mm}$. YIG thin films were deposited in high-purity oxygen with partial pressure of 5 mTorr. After deposition, the films were processed by rapid thermal annealing (RTA) at $800^{\circ} \mathrm{C}$ for $3 \mathrm{~min}$ in $\mathrm{PO} 2=2$ Torr ambient for $3 \mathrm{~min}$.

The phase identification was carried out by $\mathrm{X}$-ray diffraction (XRD) $\theta-2 \theta$ scans using a Shimatzu XRD-7000 X-ray diffractometer. The magnetic hysteresis was measured at room temperature using vibrating sample magnetometry (VSM). Fig. 1(a) shows the XRD spectrum of a YIG thin film deposited on Si substrates after rapid thermal annealing. Characteristic diffraction peaks from the garnet phase located at $2 \theta=28.9,32.4$ and 35.6 degree are observed, corresponding to the (400), (420) and (422) crystal plane diffractions of YIG. No secondary or amorphous phase is observed in films after RTA, and the broad amorphous peak located at $2 \theta \approx 20$ degree comes from the atomic oxidation layer of the substrate. The room temperature magnetic hysteresis loops of YIG/Si are given in Fig. 1(b). Both in-plane and out-of-plane hysteresis loops indicate the saturation magnetizations reaching $128 \mathrm{emu} / \mathrm{cm}^{3}$, close to the value of bulk YIG (140 emu/cm3). The films show clear in-plane anisotropy due to shape anisotropy. The coercivity is around $50 \mathrm{Oe}$, which is about ten times larger than for YIG deposited on GGG substrate. Fig. 1(c) shows a three-dimensional atomic force microscope (AFM) graph of YIG on Si substrate. The surface roughness is $0.33 \mathrm{~nm}$.

In order to get well-defined spin waves in silicon based YIG thin films, e-beam lithography and thermal evaporation are used to fabricate a rectangle pattern and CPW. A YIG stripe $3.3 \mu \mathrm{m}$ wide, 10 $\mu \mathrm{m}$ long and $100 \mathrm{~nm}$ thick was grown and a four-time meander CPW was deposited on the stripe, as it has been proven highly efficient in exciting spin waves with fixed wavelength [Vlaminck 2008]. CPW in our experiment was made of $120 \mathrm{~nm}$-thick $\mathrm{Au}$, with the width of signal lines $400 \mathrm{~nm}$ and ground lines $200 \mathrm{~nm}$, so that the microwave field distribution in nearby real space is periodic. Thanks to such a sub-micro design of CPW, spin waves with wavelength of $1 \mu \mathrm{m}$ is excited.

Alternating Current (AC) signals generated by a microwave source were applied in the CPW, with the external field perpendicular to the YIG stripe. We measured in reflection the magnetostatic surface (Damon-Eshbach mode) spin wave signal. For the purpose of improving the sensitivity of the measurement, magnetization field modulation and Lock-In Amplification were used to obtain spin wave curves. Fig. 2 gives the schematic of experiment setup.

\section{RESULTS AND ANALYSIS}

We exploit a design of meander-like CPW at sub micrometer scale as shown in Fig. 3a, which was first used in the investigation of spin wave Doppler shift effects [Vlaminck 2008]. The advantage of such CPW design is to achieve a well-defined wave vector $\mathrm{k}$ at large value. The exact $\mathrm{k}$ distribution can be calculated using the Fourier transformation of the local field excitation around the microwave antenna. Fig. 3(b) shows the results of this calculation, where we can see a pronounced main excitation at $\mathrm{k}=6.25 \mathrm{rad} / \mu \mathrm{m}$ along with some minor excitations. This $\mathrm{k}$ value implies a spin-wave wavelength $\lambda=2 \pi / \mathrm{k}=1 \mu \mathrm{m}$. Compared with conventional microwave strip lines, the excitation of spin waves is more localized and the wavelength is precisely defined by the antenna rather than by the dimension of YIG film shape [Demokritov 2006, Mosendz 2010], and therefore offers a spin wave source with well-defined wavelength for future magnonic devices, e.g. spin wave logic devices [Haldar 2016].

With such CPW spin wave antenna, we measured the spin wave reflection with a field modulation technique. Fig. 4a shows a typical field dependence resonance spectrum at $2.4 \mathrm{GHz}$. Montaya et al. [2014] showed a systematic study on different ferromagnetic resonance (FMR) measurement techniques, and found that the damping constant extracted from field modulation CPW-FMR is consistent with the one extracted from conventional cavity FMR, although the spectra appear differently. We took the full width at half maximum intensity as the linewidth and its frequency dependence is shown in Fig. 4(c).

When we consider the boundary condition of thin films for spin wave excitation and propagation, the dispersion relation for magnetostatic surface mode could be described as [Wu 2011]

$$
\omega^{2}=\omega_{0}\left(\omega_{0}+\omega_{M}\right)+\frac{\omega_{M}^{2}\left(1-e^{-2 k d}\right)}{4}
$$

where $d$ is the thickness of the thin film, $\omega_{0}=-\gamma B_{0}$ and $\omega_{M}=$ $-\gamma \mu_{0} M_{S}$. By substituting the $k$ value into the dispersion relation and comparing the fitting line with measured data (in Fig. 4(b)), the inplane saturation magnetization could be estimated. We obtained the saturation magnetization around $120 \mathrm{emu} / \mathrm{cm}^{3}$, slightly different that the value deduced from hysteresis loops for thin films. This difference may come from the e-beam lithography process.

Fig. 4(c) shows frequency dependence of the linewidth from 2.0 $\mathrm{GHz}$ to $2.8 \mathrm{GHz}$. We use this frequency dependence data to estimate the intrinsic Gilbert damping constant $\alpha$ based on:

$$
\Delta H=\Delta H_{0}+\alpha \cdot \frac{4 \pi}{\gamma} \cdot f
$$

Thus, $\alpha$ is found to be $\alpha=1.1 \times 10^{-3}$, lower than most of the metallic magnetic materials. The typical linewidth shown in this work is much smaller than the linewidth measured from an entire YIG/Si thin film. This might indicate that by patterning the sample down to micron size, we are able to reduce dramatically the contribution of two-magnon scattering in the linewidth because the effect of inhomogeneity was minimized in such YIG micro-channels. This value suggests that YIG deposited on $\mathrm{Si}$ substrate could be a promising candidate for magnon spintronics application in semiconductor engineering.

In conclusion, we excited spin waves of a well-defined wavelength in YIG micro-channel by meander coplanar waveguides. Such spin waves with small wavelength down to $1 \mu \mathrm{m}$ is essential for potential microelectronic devices based on spin waves and for future nanomagnonic devices [Grundler 2016]. PLD-grown polycrystalline YIG micro-channels on $\mathrm{Si}$ substrate show promising damping property for future magnon spintronics applications.

\section{ACKNOWLEDGMENT}

This work was supported in part by the Polish-Swiss Research Program NANOSPIN PSRP-045/2010, the Deusche Forschungsgemeinschaft SSP 1538 (SpinCat) grant no. AN762/1 and NSF China under Grant No. 11444005. Haiming Yu would like to thank Barbara Bernard for helpful discussions. 


\section{REFERENCES}

Grundler D (2015), "Reconfigurable magnonics heats up," Nature Phys., 11, 438-441, doi: 10.1038 /nphys 3349 .

Chumak A V, Vasyuchka V I, Serga A A, Hillebrands B (2015), "Magnon spintronics," Nature Phys, 11, 453-611, doi: 10.1038/nphys3347.

Yu H. Huber R, Schwarze T, Brandl F, Rapp T, Berberich P, Duerr G, Grundler D (2012), "High propagating velocity of spin waves and temperature dependent damping in a CoFeB thin film," Appl. Phys. Lett., 100, 262412, doi: 10.1063/1.4731273.

Bauer H G, Majchrak P, Kachel T, Back C H, Woltersdorf G (2015), "Nonlinear spinwave excitations at low magnetic bias fields," Nature Comms., 6, 8274, doi: $10.1038 /$ ncomms 9274 .

Linares R C, McGraw R B, Schroeder J B (1965), "Growth and properties of yttrium iron garnet single-crystal films," J. Appl. Phys., 36, 2884, doi: 10.1063/1.1714599.

Chang H, Li P, Zhang W, Liu T, Hoffmann A, Deng L, Wu M (2014), "Nanometer-thick yttrium iron garnet films with extremely low damping," IEEE Magn. Lett., 5, 6700104, doi: 10.1109/LMAG.2014.2350958.

Kelly O A, Anane A, Bernard R, Youssef J B, Hahn C, Molpeceres A H, Carrétéro C, Jacquet E, Deranlot C, Bortolotti P, Lebourgeois R, Mage J-C, Loubens G, Klein O, Cros V, Fert A. (2013), "Inverse spin Hall effect in nanometer-thick yttrium iron garnet/Pt system," Appl. Phys. Lett., 103, 082408, doi: 10.1063/1.4819157.

Bao M, Khitun A, Wu Y, Lee J-Y, Wang K L, Jacob A P (2008), “Coplanar waveguide radio frequency ferromagnetic parametric amplifier," Appl. Phys. Lett., 93, 072509, doi: 10.1063/1.2975174, doi: 10.1063/1.2975174.

Albisetti E, Petti D, Pancaldi M, Madami M, Tacchi S, Curtis J, King W P, Papp A, Csaba G, Porod W, Vavassori P, Riedo E, Bertacco R (2016), "Nanopatterning reconfigurable magnetic landscapes via thermally assisted scanning probe lithography," Nature Nanotech., 11, 545-551, doi: 10.1038/nnano.2016.25.
Cornelissen L J, Liu J, Duine R A, Ben Youssef J, van Wees B J (2015), “Long-distance transport of magnon spin information in a magnetic insulator at room temperature," Nature Phys., 11, 1022-1026, doi: 10.1038/nphys3465.

Vlaminck V, Bailleul M (2008), "Current-induced spin-wave Doppler shift," Science, 322, 410-413, doi: 10.1126/science.1162843.

Demokritov S O, Demidov V E, Dzyapko O, Melkov G A, Serga A A, Hillebrands B, Slavin A N (2006), "Bose-Einstein condensation of quasi-equilibrium magnons at room temperature under pumping," Nature, 443, 430-433, doi: 10.1038/nature05117.

Mosendz O, Vlaminck V, Pearson J E, Fradin F Y, Bauer G E W, Bader S D, Hoffmann A (2010), "Detection and quantification of inverse spin Hall effect from spin pumping in permalloy/normal metal bilayers," Phys. Rev. B, 82, 214403, doi: 10.1103/PhysRevB.82.214403.

Haldar A, Kumar D, Adeyeye A O (2016), "A reconfigurable waveguide for energyefficient transmission and local manipulation of information in a nanomagnetic device," Nature Nanotech., 11, 437-443, doi: 10.1038/nnano.2015.332.

Montoya E, McKinnon T, Zamani A, Girt E, Heinrich B (2014), "Broadband ferromagnetic resonance system and methods for ultrathin magnetic films," J. Magn. Magn. Mater., 356, 12-20, doi: 10.1016/j.jmmm.2013.12.032.

Wu M(2011), "Nonlinear spin waves in magnetic film feedback rings," Soild State Phys., vol. 62, pp. 163-224, doi: 10.1016/B978-0-12-374293-3.00003-1.

Grundler D (2016), "Spintronics: Nanomagnonics around the corner," Nature Nanotech., 11, 407-408, doi: 10.1038/nnano.2016.16. 

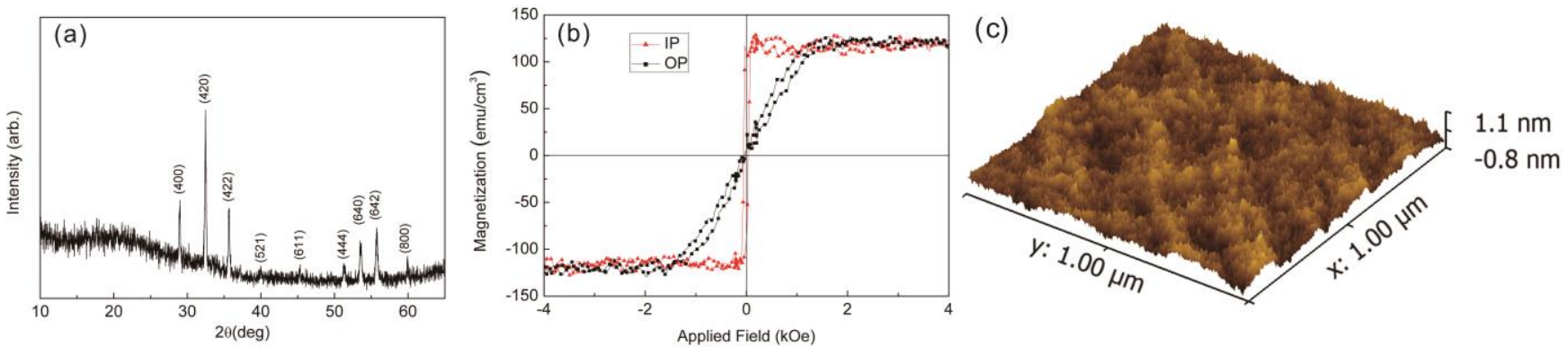

Fig. 1. (a). XRD spectrum of YIG thin films on silicon. (b). In-plane (red) and out-of plane (black) hysteresis loop of YIG thin films on silicon. (c). Three-dimensional Atomic Force Microscope graph of YIG surface.

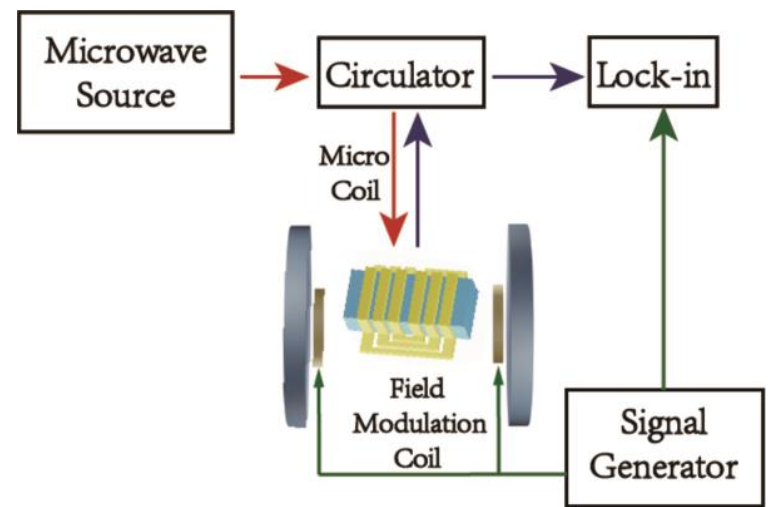

Fig. 2. Schematic of experiment setup: spin waves are excited by microwave signal generator and detected by Lock-In amplification using field modulation FMR.
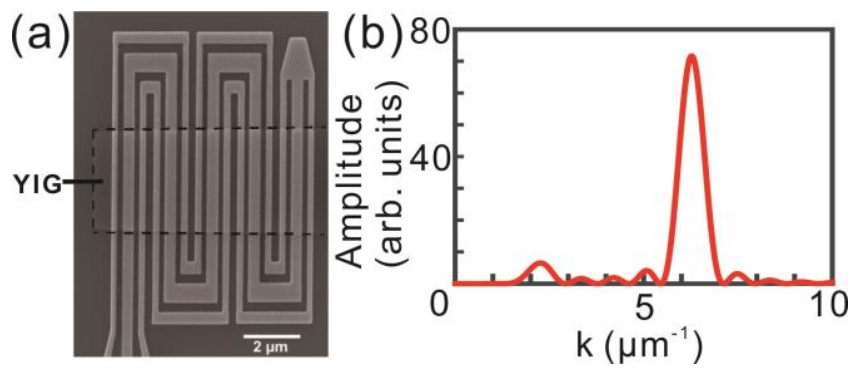

Fig. 3. (a). SEM image of meander coplanar waveguide with submicrometer scale. The width of signal line $400 \mathrm{~nm}$, ground line $200 \mathrm{~nm}$ and separation of signal line and ground line is $200 \mathrm{~nm}$. For separated units meander, the distance is $300 \mathrm{~nm}$. (b). Fourier transform of the microwave current distribution for the designed CPW in experiment.
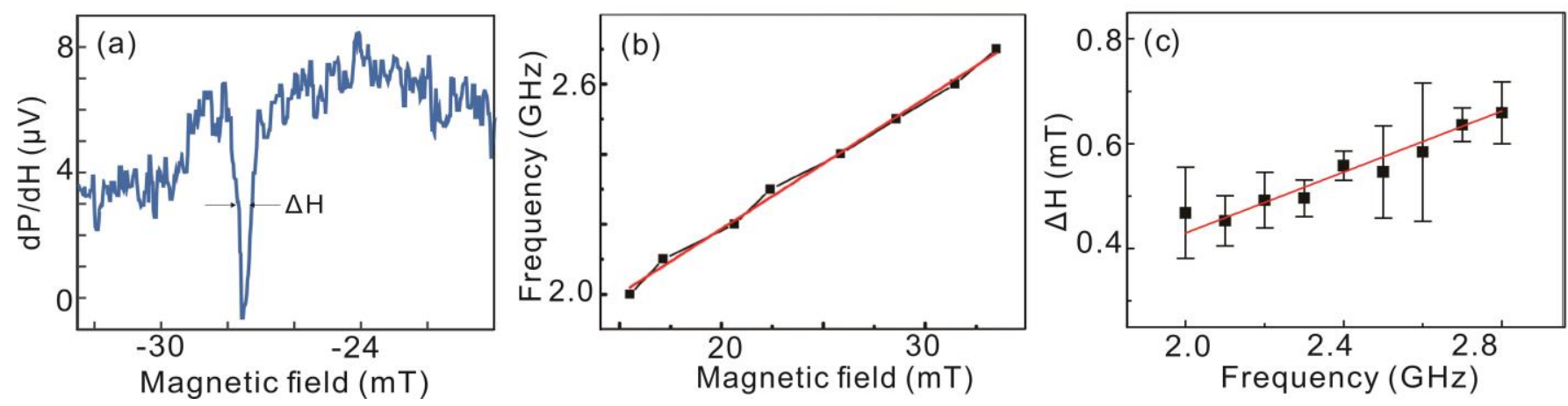

Fig. 4. (a). Reflected spin wave measured by CPW with magnetic field modulation. The linewidth is extracted using FWHM of the signal response. (b). Dispersion curve measured with CPW in silicon based YIG film. (c). Frequency dependence of FMR linewidth. Red line indicates a linear fitting where Gilbert damping parameter is extracted. Error bars indicate the noise level. 